\title{
A Multi-Center Space Data System Prototype Based on CCSDS Standards
}

\author{
Thomas M. Rich \\ NASA/Johnson Space Center \\ 2101 NASA Parkway \\ Houston, Texas 77058 \\ $281483-4984$ \\ Thomas.M.Rich-1@nasa.gov
}

\begin{abstract}
Deep space missions beyond earth orbit will require new methods of data communications in order to compensate for increasing RF propagation delay. The Consultative Committee for Space Data Systems (CCSDS) standard protocols Spacecraft Monitor \& Control (SM\&C), Asynchronous Message Service (AMS), and Delay/Disruption Tolerant Networking (DTN) provide such a method. The maturity level of this protocol set is, however, insufficient for mission inclusion at this time. This prototype is intended to provide experience which will raise the Technical Readiness Level (TRL) of these protocols.
\end{abstract}

In order to reduce costs, future missions can take advantage of these standard protocols, which will result in increased interoperability between control centers. This prototype demonstrates these capabilities by implementing a realistic space data system in which telemetry is published to control center applications at the Jet Propulsion Lab (JPL), the Marshall Space Flight Center (MSFC), and the Johnson Space Center (JSC). Reverse publishing paths for commanding from each control center are also implemented. The target vehicle consists of realistic flight computer hardware running Core Flight Software (CFS) in the integrated Power, Avionics, and Power (iPAS) Pathfinder Lab at JSC.

This prototype demonstrates a potential upgrade path for future Deep Space Network (DSN) modification, in which the automatic error recovery and communication gap compensation capabilities of DTN would be exploted. In addition, SM\&C provides architectural flexibility by allowing new service providers and consumers to be added efficiently anywhere in the network using the common interface provided by SM\&C's Message Abstraction Layer (MAL).

In FY 2015, this space data system was enhanced by adding telerobotic operations capability provided by the Robot API Deligate (RAPID) family of protocols developed at JPL. RAPID is currently being adopted as an international standard by the CCSDS Telerobotic Operations Working Group. Gateways for the purpose of interfacing RAPID messages with the existing SM\&C based infrastructure were developed. Telerobotic monitor, control, and bridge applications were written in the RAPID framework, which were then tailored to the NAO telerobotic test article hardware, a product of Aldebaran Robotics.

\section{TABLE OF CONTENTS}

1. INTRODUCTION 1

2. SPACE DATA SYSTEM OVERVIEW ......................... 2 3. SPACE DATA SYSTEM DETAILED DESIGN.............. 2 978-1-4673-7676-1/16/\$31.00 @2016 IEEE

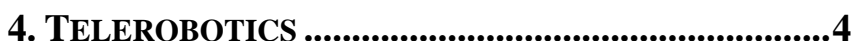

5. OBSERVATIONS .............................................................5

6. SUMMARY $\ldots \ldots \ldots \ldots \ldots \ldots \ldots \ldots \ldots \ldots \ldots \ldots \ldots \ldots \ldots \ldots \ldots \ldots \ldots \ldots \ldots \ldots \ldots \ldots \ldots \ldots \ldots . . . \ldots$

APPENDICES ......................................................................5

A. ACRONYMS ..... ERROR! BOOKMARK NOT DEFINED. ACKNOWLEDGEMENTS ........................................5

REFERENCES ......................................................5

BIOGRAPHY ...........................................................6

\section{INTRODUCTION}

Spacecraft mission control centers are typically organized by agencies and missions. Generally, each center has unique interfaces to exchange mission services and products (Telemetry, Command, Planning, Navigation, Tele- robotics, and others.) These unique interfaces drive higher costs for integration and mission cross-support.

The CCSDS Mission Operations Spacecraft Monitor and Control Working Group has been addressing these issues by developing a set of standards for interoperability. These standards are in various stages of development by several international space agencies, including NASA. As standardized interfaces are included in mission operations centers, the cost for incorporating cross support and interoperability across agencies and missions will decline.

The Space Data System prototype described in this paper demonstrates the ability to leverage services and reuse investments from several different centers on any given mission. Telemetry and Command services were implemented at the Protocol Test Lab at JPL, the Huntsville Operations Support Center (HOSC) at MSFC, and in the Operations Technology Facility (OTF) at JSC. This SM\&C and DTN-based Space Data System was originally developed in the OTF in support of the DLR Prototype ${ }^{1}$ and iPAS $^{2}$ Pathfinder activities, and was extended over DTN to support remote SM\&C applications at JPL and MSFC. 


\section{SpaCe Data System OVerview}

Figure 1 describes the general layout of this Space Data System prototype.

Telemetry in the form of CCSDS Space Packets originates with the Multi-Purpose Crew Vehicle (MPCV) vehicle system in JSC Bldg. 29. Utilizing the Licklider Transmission Protocol provided by the Interplanetary Overlay Network $(\mathrm{ION})^{3}$, the data stream is sent to a DSN Operations Center emulation in the JPL Protocol Test Lab. In the DSN Ops Center emulation, a 4 second one-way light time delay is applied, and $2 \%$ and $0.1 \%$ frame drop rates are modelled for the downlink and uplinks, respectively. The asymetric frame drop rates are due to higher power levels and larger antennae used by the ground sites. Utilizing the Asynchronous Message Service (again provided by ION) the data stream is then distributed to SM\&C consumer applications in the OTF at JSC, the HOSC at MSFC, and in the Protocol Test Lab at JPL. Command services follow the reverse paths.

Two observations can be made at this point. First, this is the only known Space Data System prototype based entirely on CCSDS international standards. Second, this is a realistic prototype for potential future DSN automation based on Delay/Disruption Tolerant Networking.

\section{SPaCe Data System Detailed Design}

Figure 2 describes the Space Data System detailed design.

A description of each segment follows.

\section{Flight Computer Segment}

Core Flight Software from the Goddard Space Flight Center runs on representative flight-like hardware in iPAS vehicle Bay 2, JSC Bldg. 29. An environment simulation generates sensor data, which is telemetered through the flight computer to the space-to-ground communications processor known as "datapub2." The iPAS avionics suite contains an onboard power system, which processes commands relayed through the flight computer. A muffin fan is configured as the command target. Both telemetry and command data are formatted as CCSDS Space Packets, sent between "datapub2" and the flight computer over the User Datagram Protocol (UDP).

\section{Onboard Communications Processor Segment "datapub2"}

datapub2 hosts the onboard SM\&C provider and Delay / Disruption Tolerant Networking processes. The SM\&C Parameter Provider process ingests the CCSDS Space Packet telemetry stream from the iPAS flight computer. It then passes SM\&C messages to the DTN process using the Asynchronous Message Service, which provides publish and subscribe functionality. AMS messages are then sent to the DSN Ops Center emulation at JPL using the Licklider

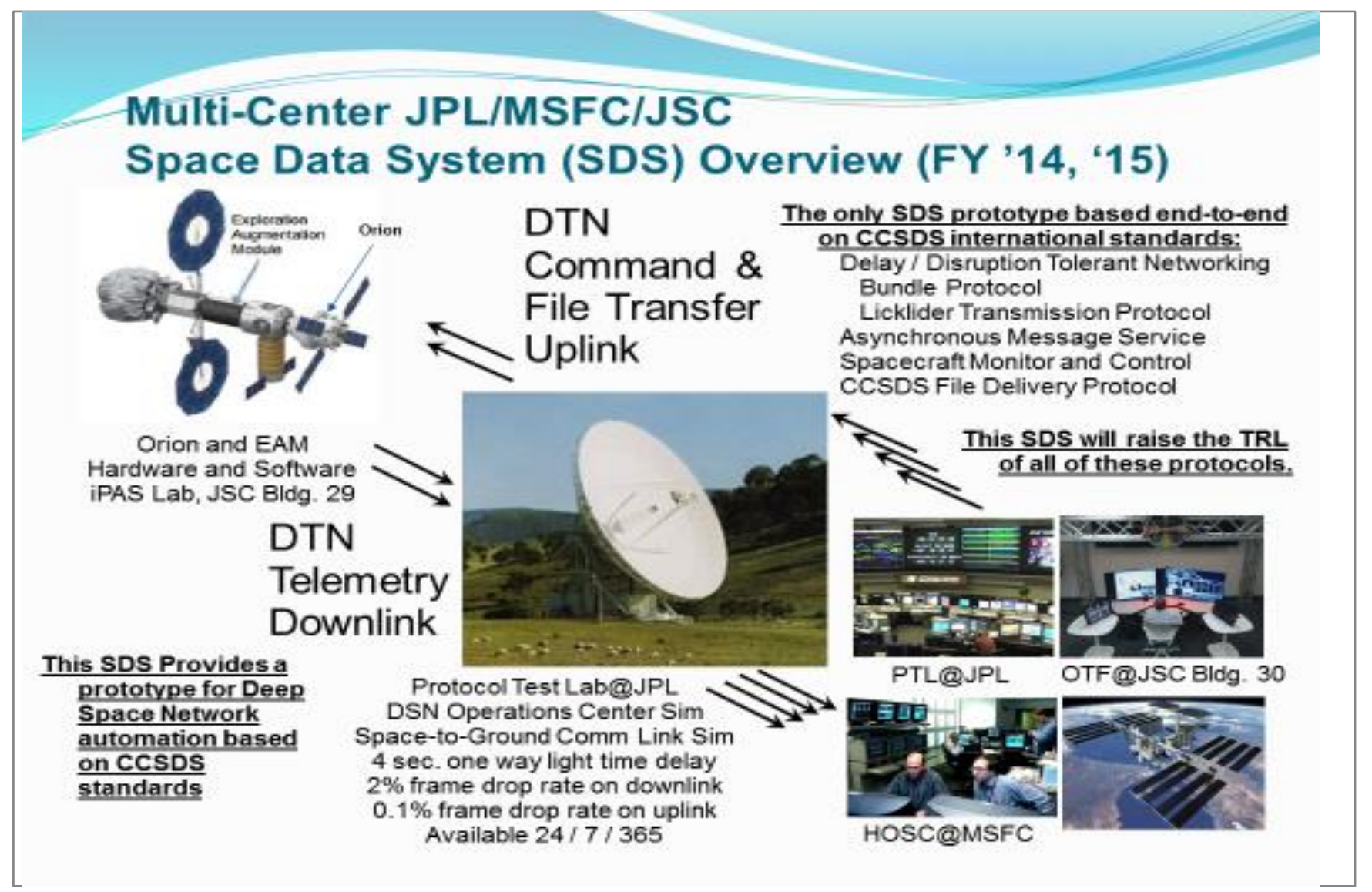




\section{Multi-Control-Center Space Data System}
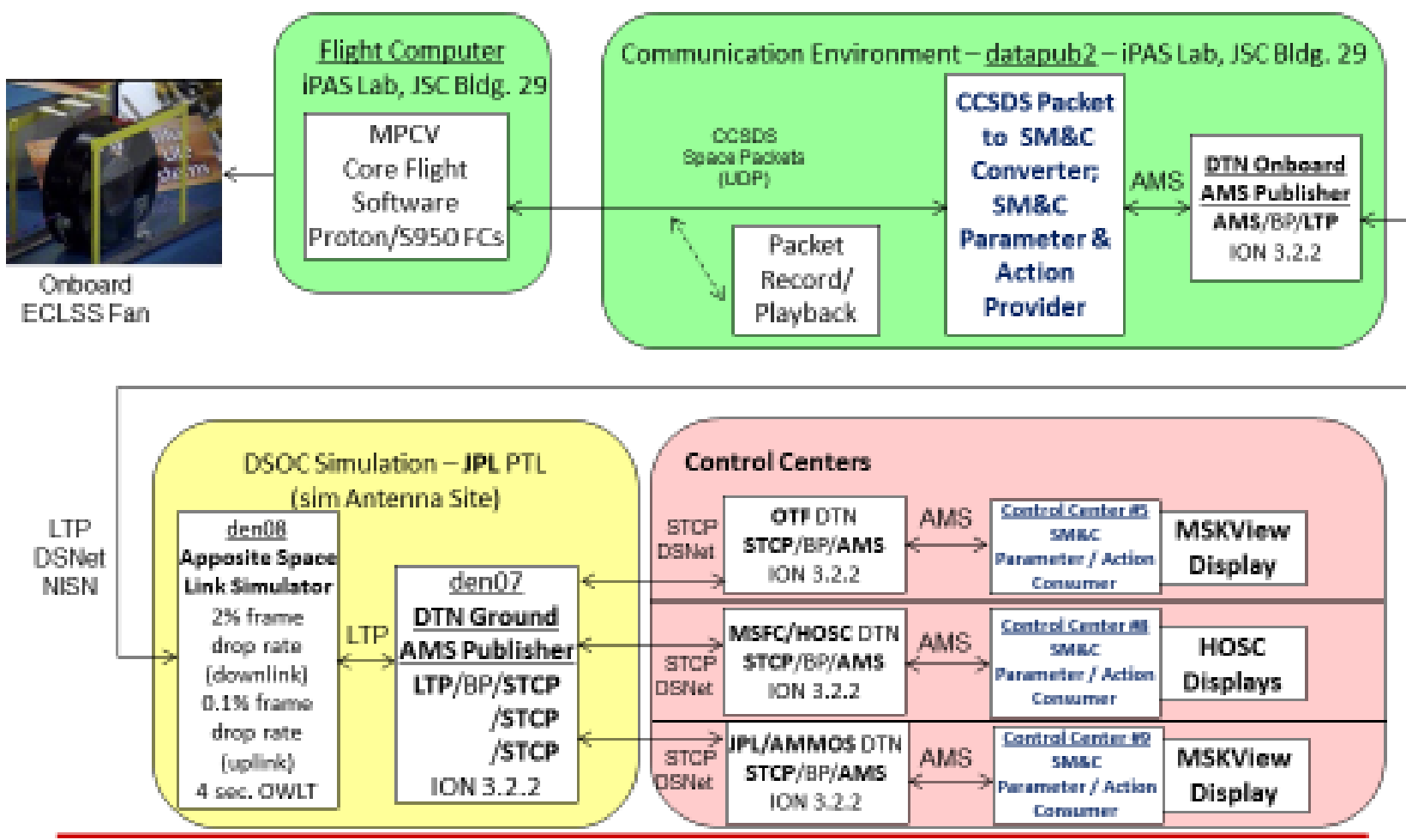

Project Exit Presentation

Transmission Protocol (LTP). LTP is a reliable connectionless protocol which provides automatic error detection and re-transmission. LTP is designed to support RF propagation delays on the order of interplanetary distances. The NASA Institutional Services Network (NISN) provides terrestrial connectivity.

datapub2 also hosts the SM\&C Action Provider, which receives command messages through the AMS / DTN ground-to-space link. The Action Provider generates CCSDS Space Packet command packets and forwards them to the flight computer for final relay to the onboard power system and the ECLSS fan.

\section{DSN Operations Center Emulation Segmemt}

Two main functions are implemented at the DSN Operations Center emulation in the Protocol Test Lab at JPL. The first is the space-to-ground link model, which applies a 4 second one-way-light-time delay, a $2 \%$ frame error rate on the downlink, and a $0.1 \%$ frame drop rate on the uplink.

The second main function of the DSOC emulator is to anchor the ground side of the AMS / LTP / DTN link, and to then republish the AMS telemetry streams for distribution to geographically dispersed control centers. Both Simple Transmission Control Protocol (STCP) and datagram re- transmission are used to provide reliable terrestrial links. Terrestrial links over UDP are also supported.

\section{Control Center Segment}

The original set of SM\&C control center applications was developed in the OTF. The applications interface directly with the DSOC distribution node at JPL. The applications are used to display telemetry and command history, as well as initiating commands to control the onboard ECLSS fan.

These applications, as well as the SM\&C interface software, were then sent to the Protocol Test Lab at JPL, where they were compiled and installed. Telemetry display and command functionality are transparent between the PTL and OTF control center applications. Telemetry displays are identical at both control centers, as well as command capability.

The same SM\&C interface software was then sent to the HOSC at MSFC. In order to display telemetry, the HOSC "Display Dashboard" application was fitted with the SM\&C common interface. The capability to easily add new parameters to the telemetry display was demonstrated. A new SM\&C command application was developed, in order to generate ECLSS fan commands. Lines of text should be single-spaced, except for double spacing before headings and space-and-a-half after. 


\section{Telerobotic Multi-Control-Center Architecture (FY '15)}

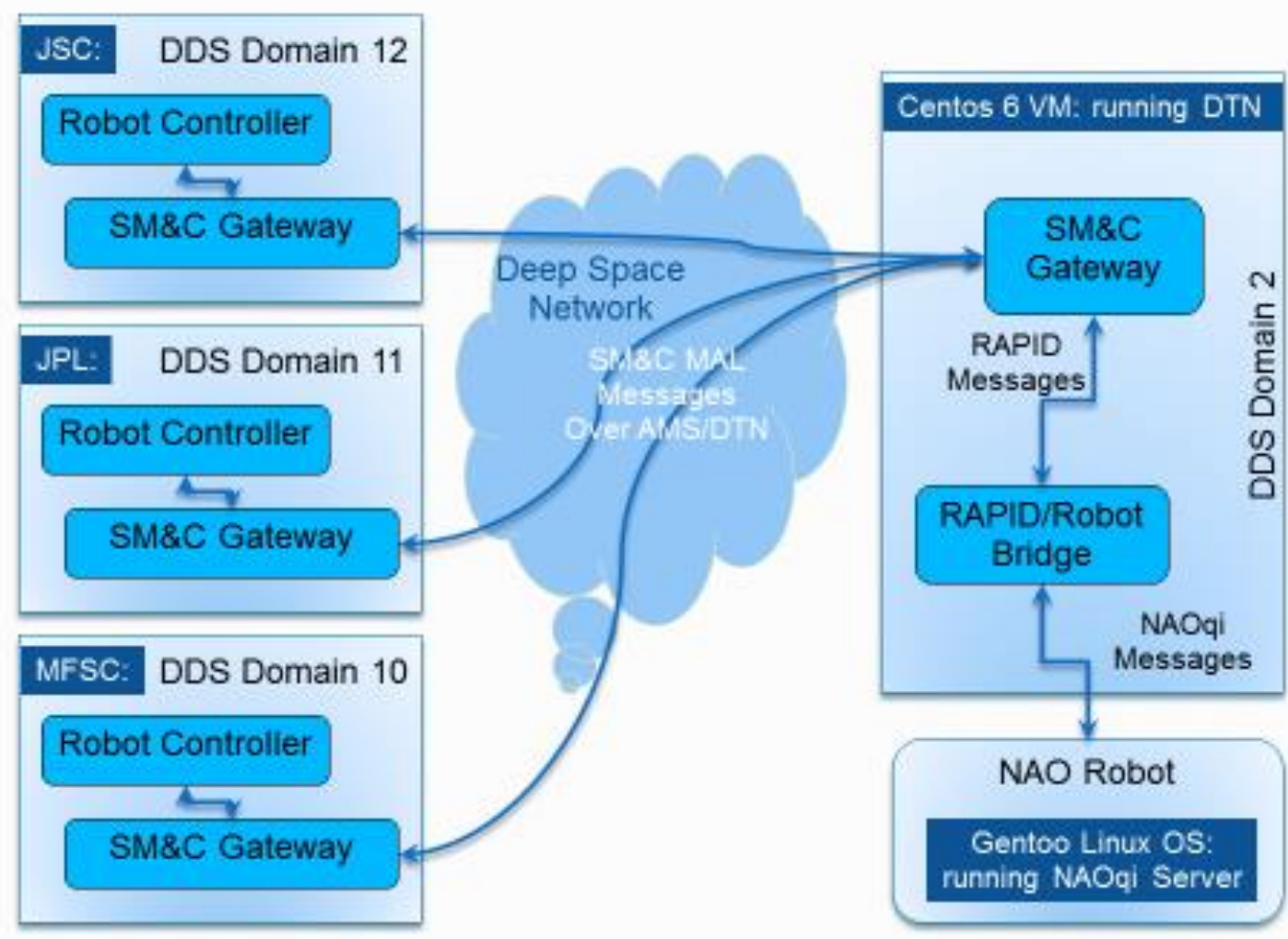

\section{TELEROBOTICS}

Figure 3 describes the multi-center telerobotic control architecture.

A description of each segment follows.

\section{Robot Controller Segments}

The robot controller software module was developed in the Eclipse development environment, modified by JPL to include the Robot API Delegate (RAPID) telerobotic monitor and control protocols. The controller consists of views of robot joints and temperatures, battery charge level, onboard camera views. The controller has the ability to command each joint separately, and to activate pre-programmed behaviors and postures. The controller includes the RAPID Access Control module, which is used to coordinate robot control authority between multiple centers. As Figure 3 shows, multiple instances of the robot controller can be executed simultaneously.

The Eclipse / RAPID development environment uses Data Distribution Service (DDS) as its transport layer. RAPID commands and telemetry are expressed as DDS messages.

\section{SM\&C Controller Gateway Segments}

The SM\&C controller gateway segments were introduced in order to interface the DDS based RAPID messages with the SM\&C based Multi-Center Space Data System. The controller gateway simply encapsulates DDS messages into $\mathrm{SM} \& \mathrm{C}$ messages.

\section{Deep Space Network Segment}

The DSN segment is the Multi-Center Space Data System described in sections II and III.

\section{SM\&C Bridge Gateway Segments}

The SM\&C bridge gateway extracts DDS messages from the SM\&C messages transported over the DSN segment.

\section{The RAPID / Robot Bridge Segment}

The RAPID / robot bridge segment performs the final step of converting RAPID standard messages (over DDS) into robot specific commands, using the software development kit provided by the robot manufacturer. 


\section{OBSERVATIONS}

This prototype is end-to-end, in that SM\&C and AMS processes are executed onboard, as well as the ground. This has an impact on both onboard software complexity and flight computer resource utilization. A hybrid configuration is possible in which SM\&C and AMS processes are restricted to ground resources.

This "all SM\&C" configuration offers the advantage of architectural flexibility, in which SM\&C consumer and provider processes can be located anywhere in the network. This has been demonstrated in the iPAS, in which identical consumer processes have been executed both onboard and on the ground.

This prototype Space Data System has run continuously over the past two years. As the software has matured, system stability has improved. Unplanned outages have become rare.

\section{SuMmary}

This project has shown that space mission information can be distributed to multiple control centers using standard Spacecraft Monitor and Control services. At the control center endpoints, local applications can then be easily integrated into the SM\&C and DTN infrastructure. When standard protocols such as SM\&C and DTN are utilized, interoperability increases, and both per-mission and control center costs decrease. Since these are internationally standardized protocols, international interoperability can increase as well.

Interaction with the iPAS Pathfinder Lab has provided the opportunity to operate this multi-center Space Data System in a realistic scenario where light-time delay becomes significant. iPAS visibility has provided the opportunity to promote the acceptance and application of these internationally standardized protocols. This will contribute to raising their Technical Readiness Levels, and eventual incorporation into mission architectures and Deep Space Network re-design.

\section{APPENDICES}

\section{A. ACronyms}

$\begin{array}{ll}\text { CCSDS } & =\text { Consultative Committee for space Data Systems } \\ \text { CFS } & =\text { Core Flight software } \\ \text { DDS } & =\text { Data Distribution Service } \\ \text { DSN } & =\text { Deep Space Network } \\ \text { DTN } & =\text { Delay / Disruption Tolerant Networking } \\ \text { GSFC } & =\text { Goddard Space Flight Center } \\ \text { HOSC } & =\text { Huntsville Operations Support Center } \\ \text { ION } & =\text { Interplanetary Overlay Network } \\ \text { iPAS } & =\text { integrated Power, Avionics, and Software } \\ \text { JSC } & =\text { Johnson Space Center } \\ \text { JPL } & =\text { Jet Propulsion Laboratory } \\ \text { LTP } & =\text { Licklider Transmission Protocol }\end{array}$

MAL = Message Abstraction Layer

MSFC = Marshall Space Flight Center

NASA = National Aeronautics and Space Administration

NISN = NASA Institutional Services Network

OTF = Operations Technology Facility

RAPID $=$ Robot API Delegate

PTL = Protocol Test Lab

RF $\quad=$ Radio Frequency

$\mathrm{SM} \& \mathrm{C}=$ Spacecraft Monitor and Control

TCP = Transmission Control Protocol

TRL = Technical Readiness Level

UDP = User Datagram Protocol

\section{ACKNOWLEDGEMENTS}

This paper summarizes the achievements of the Multi-Center Space Data System Prototype team. The author wishes to acknowledge several individuals for their contributions. Pete Gonzalez/JSC provided the software engineering for the project; without his understanding of SM\&C and the RAPID telerobotics protocol this project would not have been possible. Leigh Torgerson/JPL provided the space networking expertise for the DSN Operations Center emulation at JPL. Pat Donahue/MSFC provided the software engineering for the HOSC applications. Eric Wolfer/JSC and Jay Wyatt/JPL provided project management and funding. And finally, the late Lindolfo Martinez provided overall vision and guidance for the project.

\section{REFERENCES}

[1] Lucord, S., "CCSDS SM\&C Mission Operations Interoperability Prototype," AIAA-2010-2338, SpaceOps 2010, Huntsville AL, 25-30 April 2010

[2] Othon, W., "iPAS: AES Flight Systems Technology Maturation for Human Spaceflight," AIAA-2014-1925, SpaceOps 2014, Pasadena CA, 5-8 May 2014

[3] Burleigh, S., ION.pdf, distributed with ION open source 


\section{BIOGRAPHY}

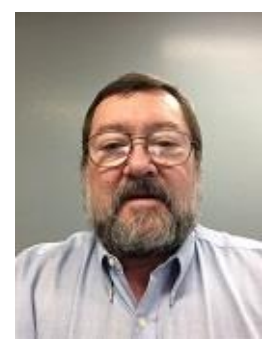

Thomas M. Rich received a BSEE and an MEE in Electrical Engineering from Rice University, Houston in 1973 and 1974. He has been with contractor companies at JSC for more than 41 years, all in support of Space Shuttle flight control and flight planning systems in JSC Building 30, home of the Space Shuttle and International Space Station Mission Control Centers. He is currently the lead for the advanced spacecraft communication prototyping effort in the Operations Technology Facility, Mission Systems Division, JSC. Prior to the OTF, he served as Integrated Planning System system engineer for United Space Alliance. He was a certified navigation flight controller for STS-1, the first flight of the Space Shuttle Program. His career started with developing onboard and ground based navigation software for the Space Shuttle program for McDonnell Douglas. He is presently a Senior System Engineer with Tietronix Software. 
